\title{
BMJ Open Study protocol on criterion validation of Edinburgh Postnatal Depression Scale (EPDS), Patient Health Questionnaire (PHQ-9) and Centre for Epidemiological Studies-Depression (CES-D) screening tools among rural postnatal women; a cross-sectional study
}

To cite: January J, Chimbari MJ. Study protocol on criterion validation of Edinburgh Postnatal Depression Scale (EPDS), Patient Health Questionnaire (PHQ-9) and Centre for Epidemiological Studies-Depression (CES-D) screening tools among rural postnatal women; a crosssectional study. BMJ Open 2018;8:e019085. doi:10.1136/ bmjopen-2017-019085

- Prepublication history for this paper is available online. To view these files, please visit the journal online (http://dx.doi. org/10.1136/bmjopen-2017019085).

Received 10 August 2017 Revised 5 February 2018 Accepted 7 February 2018

D) Check for updates

${ }^{1}$ Department of Psychiatry, School of Clinical Medicine, College of Health Sciences, University of KwaZulu-Natal, Durban, South Africa

${ }^{2}$ School of Nursing and Public Health, College of Health Sciences, University of KwaZuluNatal, Durban, South Africa

Correspondence to

James January;

miranda.january@gmail.com

\section{ABSTRACT}

Background Screening women for postnatal depression (PND) provides an opportunity to reach undetected cases and enhance pregnancy outcomes. In Zimbabwe, no validation of depression screening tools has been done on postnatal women in rural settings.

Objectives This study aims to determine criterion validity of the Edinburgh Postnatal Depression Scale (EPDS), the Patient Health Questionnaire (PHQ-9) and the Center for Epidemiological Studies-Depression (CES-D) scale using the Diagnostic and Statistical Manual for Mental Disorders (DSM-5) criteria as the reference standard.

Methods Women $(n=462)$ attending postnatal care at 7 or 42 days at two rural district hospitals in Zimbabwe will be assessed for depressive symptoms using the EPDS, PHQ-9 and CES-D. The women will be interviewed by a clinical psychologist using DSM- 5 criteria. Sensitivities, specificities, positive predictive values, negative predictive values and test efficiencies will be calculated for each of the three tools. The area under the receiver operating curve will quantify the overall ability of the three tests to discriminate between those mothers with PND and those without.

Discussion Findings from this study will add to the body of knowledge on PND among women in resourcelimited settings. Identifying women with PND will enable healthcare providers to link them with care, which will ultimately improve maternal and child health outcomes. Furthermore, this study will provide evidence on which screening tool would be best for screening PND in rural settings of Zimbabwe.

\section{INTRODUCTION}

Depression during the perinatal period is a major cause of morbidity and mortality. ${ }^{1}$ Postnatal depression (PND) has adverse longterm ramifications on women, their children and communities. Recently, the United States Preventive Services Task Force recommended screening of women for depressive symptoms in primary healthcare settings during the perinatal period as screening has the potential to substantially enhance accurate identification of those at risk, ${ }^{2}$ thereby necessitating timely interventions. In support of such recommendations, evidence from a recently completed systematic review shows that depression screening programmes can significantly reduce the risk of depression among pregnant and postpartum women. ${ }^{3}$

The global prevalence of PND ranges

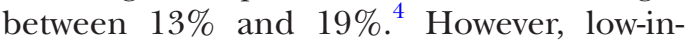
come and middle-income countries are disproportionately affected with prevalence $>30 \%$ in Zimbabwe, ${ }^{5-7} \mathrm{Nepal}^{8}$ rural South India $^{9}$ and South Africa. ${ }^{10}$ Despite these high prevalences of PND in Zimbabwe, it is rarely diagnosed or managed in primary care settings. Research conducted on prevalence and correlates of PND in the country have largely been carried out in urban areas 561112 and data on periurban and rural populations are sparse. ${ }^{7}$ In South Africa, high prevalences $(50.3 \%)$ of PND among rural women have been reported. ${ }^{10}$ Thus, there is need for studies to find out if women in rural Zimbabwe are similarly affected by PND.

In Zimbabwe, screening women for depression during the perinatal period is not routinely done despite evidence which suggests that it may be beneficial in reducing depressive symptoms. ${ }^{3}$ This is in part due to there being no policy to screen for depression during the perinatal period. Additionally, there is limited training among primary healthcare providers on use of screening 
tools. Problems arise when screening for PND is done using different tools, which have different psychometric properties. If screening for depressive symptoms is to be integrated in primary care in Zimbabwe, there would be need to identify the most appropriate tool and cut-off to use as previous studies have used tools with different cut-off scores. ${ }^{13}$ Although there have been previous attempts to validate the Shona version of the Edinburgh Postnatal Depression Scale (EPDS) in Zimbabwe, ${ }^{14}$ the extent of its utility is limited to urban women. Similarly, the Patient Health Questionnaire (PHQ-9) has recently been validated among urban primary care attenders ${ }^{15}$ in Harare. The Center for Epidemiological Studies-Depression (CES-D) has not yet been validated in the country, although previous studies have employed it to estimate prevalence of depressive symptoms among women. Thus, there is need to validate these three screening tools and establish their concordance with each other so as to determine which one would be ideal to use in screening for depressive symptoms in primary care settings. Since there usually are no mental health specialists at primary care level in Zimbabwe, screened women could be referred to lay health counsellors who have been shown to be effective in delivering brief psychological interventions in primary care settings within Harare. ${ }^{16}$ In light of the foregoing arguments, this study aims to validate the EPDS, PHQ-9 and CES-D among postnatal women in a rural population in Zimbabwe.

\section{METHODS \\ Design and setting}

A cross-sectional design will be used to address the objectives of this study. The study will be conducted in selected rural health centres within Chipinge and Mutasa Districts in Manicaland Province, Zimbabwe. Manicaland has seven districts and is one of the most burdened provinces in the country regarding maternal and child mortality. The province has the highest infant mortality rate in Zimbabwe according to the 2010/2011 Demographic Health Survey. Manicaland Province also has the lowest percentage $(86.4 \%)$ of antenatal care from a skilled healthcare provider and the second lowest postnatal care in the first 2 days of life. ${ }^{17}$ Data collection will be done at major public health centres within the districts, namely Chipinge District Hospital and Mt. Selinda Hospital in Chipinge and Hauna, Bonda and Old Mutare Hospitals in Mutasa.

\section{Participants}

Consecutive women attending postnatal services at district hospitals in Chipinge and Mutasa will be recruited into the study. All women attending PNC at 7 days and 6 weeks postdelivery will be eligible to take part in the study. We will include mothers who are aged 16 years and above. The reason for including mothers below 18 years (emancipated minors) stems from the evidence that shows that younger mothers tend to have higher rates of
PND and also have lower social support. ${ }^{18}$ Women with psychosis as determined by a resident medical doctor or psychiatric nurse prior to the study being conducted and those who will be seriously ill and unable to freely give written consent will be excluded from the study. The sample size was calculated using the Dobson formula assuming a prevalence of PND among women in rural areas to be $50 \%$ (this assumes maximum variability and will yield the largest sample size) as there are no studies that have measured the prevalence in this population in Zimbabwe. We used the Dobson formula since this validation is part of a larger cross-sectional study aimed at determining determinants of postnatal depression among women in rural Manicaland. Thus, the calculated sample size obtained was adjusted to 462 assuming a $20 \%$ non-response as we anticipate that some women may not complete the entire assessment as they travel long distances to the health facility which may result in them desiring to go back home early as women will have to complete the assessment on the day they will be receiving postnatal care services.

\section{Procedures}

Consenting women will be asked to fill out a questionnaire capturing their sociodemographic details. Three trained research assistants who will be locally based in the districts and possess degree level qualifications in public health will individually approach eligible women. The research assistants will obtain written consent, collect sociodemographic information and administer already existing and unmodified Shona language versions of the EPDS, PHQ-9 and CES-D screening tools in a private room at the health facility. Women will then be referred to a clinical psychologist who will administer an interview based on the Diagnostic and Statistical Manual for Mental Disorders (DSM-5) criteria on the same day. The psychologist will be blinded to respondents' scores on the EPDS, CES-D and PHQ-9. Women meeting criteria for depression will then be further assessed for treatment and referred to doctors or psychiatric nurses stationed at the hospitals for further management.

\section{Measures}

\section{Edinburgh Postnatal Depression Scale}

The EPDS is a 10-item, widely used screening scale for antepartum and postpartum depression. The validated Shona translation of the EPDS has previously been used in studies within Zimbabwe. ${ }^{5-7}$ To ascertain whether one has depression or not, scores on each of the 10 items are added up giving a minimum score of 0 and a maximum of 30 . A cut-off of $11 / 12$ is usually used, with those scoring below the cut-off being classified as not having depression. A higher score denotes more of the depressive symptoms one would be experiencing. The items on this screening tool correspond to depressive symptoms such as anhedonia, sleep disturbances, lethargy and feelings of guilt. The sensitivity and specificity of the validated 
Shona version of the EPDS have been reported to be $88 \%$ and $87 \%$, respectively based on a cut-off score of $11 / 12 .{ }^{14}$ Additionally, the positive predictive value and negative predictive values of the tool were reported to be $74 \%$ and $94 \%$, respectively making it a reliable and valid screening tool for PND in urban settings. ${ }^{14}$

\section{Patient Health Questionnaire}

The PHQ-9 is the preferred screening tool for depressive symptoms in most primary care settings ${ }^{19}$ with estimations of accurately identifying true cases being $80 \%$. The PHQ-9 is a multipurpose self-rating nine-item, depression-screening scale, which assesses the experiencing of depressive symptoms over the last 14 days. ${ }^{20}$ Scores on the PHQ-9 range from 0 to 27 and are calculated by assigning scores of 0,1 , 2 or 3 to response categories of 'not at all', 'several days', 'more than half the days' or 'nearly every day', respectively and then summing up the scores. Greater scores on the PHQ-9 indicate more severe depressive symptoms. In a recent validation study of screening tools for depressive and anxiety disorders among patients attending primary care services in Harare, Chibanda et al reported that the sensitivity of the PHQ-9 was $85 \%$ (95\% CI $78 \%$ to 90\%) and specificity was $69 \%$ (95\% CI $59 \%$ to $77 \%$ ) based on an optimal cut-off of $\geq 11$. $^{15}$

\section{Centre for Epidemiological Studies-Depression Score}

The 20-item CES-D has been used extensively in Africa to screen for PND. Single items are scored $0,1,2$ or 3 depending on the frequency of symptoms (rarely, some time, occasionally or all of time). The total is obtained by summing up scores on the individual 20 items. Scores range from 0 to 60 . Scores of 16 or above are usually used to indicate risk for clinical depression. Recently, a Shona version was used to assess for PND among women and its association with experiencing of intimate partner violence in Zimbabwe. ${ }^{12}$ Evidence from Uganda suggests that the tool is reliable and valid for use among women attending antenatal care. ${ }^{21}$ No previous validation of the CES-D has been done in Zimbabwe.

\section{DSM-5 interview}

Diagnosis of current major depression will be based on DSM-5 criteria, which entail that five or more of the following symptoms have to be present nearly every day during a 2-week period: a) depressed mood most of the day nearly every day, b) anhedonia or markedly decreased/loss of interest or pleasure in almost all activities, c) clinically significant weight loss or increase or decrease in appetite, d) insomnia or hypersomnia, e) psychomotor agitation or retardation, f) fatigue or loss of energy, g) feelings of worthlessness or excessive or inappropriate guilt, $\mathrm{h}$ ) diminished ability to think or concentrate, or indecisiveness and i) recurrent thoughts of death or suicidal ideation. For a diagnosis of depression to be made, there should be (a) depressed mood or (b) loss of interest or pleasure, (c) impairment of social, occupational or educational function and (d) significant clinical distress in the individual.

\section{Data analysis}

Quantitative data will be analysed using descriptive and inferential statistics. Prevalence of PND will be determined using the DSM-5 criteria; and will be compared across the two districts. The performance of EPDS, CES-D and PHQ-9 against the DSM- 5 criteria will be measured. For each screening tool, the sensitivity, specificity, positive predictive value and negative predictive value for different cut-off points will be determined. Cut-off points will be chosen to provide a good balance of sensitivity and specificity and will be determined using the Youden Index, which summarises the performance of a test and ranges from -1 to 1 . The cut-off point with the greatest Youden Index will be used as the optimal. Sensitivity (true positive rate) against specificity (false positive rate) will be presented on receiver operating curves (ROC). The area under the ROC curve (AUROC) will quantify the overall ability of the three tests to discriminate between mothers with PND and those without. Thus, an AUROC of 0.8 or above will be considered as good in this study. The DeLong Method will be used to compare the AUROCs across the three screening tools. ${ }^{22}$ Furthermore, concordance and agreement across the EPDS, PHQ-9 and CES-D will be determined using the Cohen's Kappa statistic. The internal consistency of the screening tools will be determined using Cronbach's alpha. Performance of the three measures between the 7 and 42 days will be assessed.

\section{Ethics and dissemination}

Only women who will provide written informed consent will be recruited into the study. Emancipated minors will also be required to provide written consent of their guardians. The nature of this study may cause some discomfort to women as they may recollect experiences of past adverse events. Care will be taken to link all the women likely to experience distress during the study to appropriate psychological care. Explanations will be given to women that the topic under study may evoke emotions and as such they would be assured that they would be free to terminate the interview at any stage without penalty or prejudice. Since there are no psychiatrists and/or clinical psychologists in the two districts, all women who will be found to have severe depression or suicidal thoughts will be referred to doctors or psychiatric nurses stationed at the hospitals where the women will be seeking postnatal services. In addition, the Zimbabwe National Association for Mental Health has pledged to offer their Manicaland-based staff to facilitate psychological help to affected women. Results of the study will be published in a peer-reviewed journal and shared with the Ministry of Health, Manicaland, Zimbabwe at study completion in 2018.

\section{DISCUSSION}

The aim of this study is to establish criterion validity of three depression screening tools namely the EPDS, PHQ-9 and CES-D among women attending postnatal services in rural settings within a resource-constrained 
setting. Such a study is timely as it adds to the body of knowledge on PND among women in resource-limited settings. Identifying women with PND will enable healthcare providers to link them with appropriate care, which will ultimately improve maternal and child health outcomes. Furthermore, this study will provide evidence on which screening tool would be best to use in rural settings within Zimbabwe to screen for PND. To the authors' knowledge, this is the first study to validate the three depression screening tools among women in rural health settings in Zimbabwe. In light of the high prevalence of PND that has been previously documented in Zimbabwe, ${ }^{5-7}$ findings from this study can be compared with those from other settings so as to ascertain whether parallels exist.

In summary, there is paucity of data on the utility of the EPDS, PHQ-9 and CES-D screening tools for PND among women in rural settings in Zimbabwe. ${ }^{13}$ In order to fully integrate mental health into maternal health services, there is need to routinely screen women during the antenatal and postnatal periods so that appropriate and timely interventions can be instituted.

There are some limitations in the proposed study. First, the study will be health facility based and will thus miss out those women who may not be reporting to healthcare facilities. In future, efforts should be made to explore the feasibility of community screening for PND. Second, the study will focus only measuring criterion validity of the three tools. Given that the CES-D has not been previously validated in Zimbabwe, there is need for future studies to measure the construct validity to ensure that the items are locally understandable. Third, random order effects will not be assessed as the administration of the three screening tools will not be varied and all interviews conducted on the same day. Due to limited resources, the study will use only one clinical psychologist to administer the interview thus potentially introducing assessment bias and limit reliability and validity of the assessment. In order to minimise this, the psychologist administering the DSM-5-based interview will be blinded to results from the EPDS, PHQ-9 and CES-D. Lastly, our results may not necessarily extrapolate to other women within rural settings in Zimbabwe due to cultural and socioeconomic variations.

Contributors $\mathrm{JJ}$ conceived the study. Both $\mathrm{JJ}$ and MJC wrote the first and subsequent drafts of the manuscript. Both authors read and approved the final manuscript.

Funding This study is supported by the University of KwaZulu-Natal College of Health Sciences PhD scholarship fund awarded to JJ for the year 2017.

Disclaimer The funders of the scholarship had no role in the design of the study, collection, analysis, interpretation of data and in writing the manuscript.

Competing interests None declared.

Patient consent Not required.

Ethics approval The permission to proceed has been granted by the Zimbabwean Ministry of Health and Child Care at national, provincial, district and health facility levels. The research protocols have been reviewed and approved by the institutional review boards of the University of KwaZulu-Natal UKZN Biomedical Research Ethics Committee (BE598/16), the Joint Research Ethics Committee for the University of
Zimbabwe, College of Health Sciences and the Parirenyatwa Group of Hospitals (JREC/30/17) and the Medical Research Council of Zimbabwe (MRCZ/A/2161). Medical Research Council of Zimbabwe.

Provenance and peer review Not commissioned; externally peer reviewed.

Open Access This is an Open Access article distributed in accordance with the Creative Commons Attribution Non Commercial (CC BY-NC 4.0) license, which permits others to distribute, remix, adapt, build upon this work non-commercially, and license their derivative works on different terms, provided the original work is properly cited and the use is non-commercial. See: http://creativecommons.org/ licenses/by-nc/4.0/

(C) Article author(s) (or their employer(s) unless otherwise stated in the text of the article) 2018. All rights reserved. No commercial use is permitted unless otherwise expressly granted.

\section{REFERENCES}

1. Ramadas S, Kumar R. Postnatal depression: a narrative review. Int $J$ Cult Ment Health 2016;9:97-107.

2. Siu AL, Bibbins-Domingo K, Grossman DC, et al. Screening for depression in adults: US preventive services task force recommendation statement. JAMA 2016;315:380-7.

3. O'Connor E, Rossom RC, Henninger M, et al. Primary care screening for and treatment of depression in pregnant and postpartum women: evidence report and systematic review for the us preventive services task force. JAMA 2016;315:388-406.

4. O'Hara MW, McCabe JE. Postpartum depression: current status and future directions. Annu Rev Clin Psychol 2013;9:379-407.

5. Chibanda D, Mangezi W, Tshimanga M, et al. Postnatal Depression by HIV Status Among Women in Zimbabwe. J Womens Health 2010;19:2071-7.

6. January J, Chivanhu $\mathrm{H}$, Chiwara J, et al. Prevalence and the correlates of postnatal depression in an urban high density suburb of Harare. Cent Afr J Med 2015;61-1-4.

7. January J, Mutamba N, Maradzika J. Correlates of postnatal depression among women in Zimbabwean semi-urban and rural settings. J Psychol Afr 2017;27:93-6.

8. Giri RK, Khatri RB, Mishra SR, et al. Prevalence and factors associated with depressive symptoms among post-partum mothers in Nepal. BMC Res Notes 2015;8:111.

9. Shivalli S, Gururaj N. Postnatal depression among rural women in South India: do socio-demographic, obstetric and pregnancy outcome have a role to play? PLoS One 2015;10:e0122079.

10. Stellenberg EL, Abrahams JM. Prevalence of and factors influencing postnatal depression in a rural community in South Africa. Afr J Prim Health Care Fam Med 2015;7:874.

11. Nhiwatiwa S, Patel V, Acuda W. Predicting postnatal mental disorder with a screening questionnaire: a prospective cohort study from Zimbabwe. J Epidemiol Community Health 1998;52:262-6.

12. Shamu S, Zarowsky C, Roelens K, et al. High-frequency intimate partner violence during pregnancy, postnatal depression and suicidal tendencies in Harare, Zimbabwe. Gen Hosp Psychiatry 2016;38:109-14.

13. January J, Burns J, Chimbari M. Primary care screening and risk factors for postnatal depression in zimbabwe: a scoping review of literature. J Psychol Afr 2017;27:294-8.

14. Chibanda D, Mangezi W, Tshimanga M, et al. Validation of the edinburgh postnatal depression scale among women in a high HIV prevalence area in urban Zimbabwe. Arch Womens Ment Health 2010;13:201-6.

15. Chibanda D, Verhey R, Gibson LJ, et al. Validation of screening tools for depression and anxiety disorders in a primary care population with high HIV prevalence in Zimbabwe. J Affect Disord 2016;198:50-5

16. Abas M, Bowers T, Manda E, et al. 'Opening up the mind': problemsolving therapy delivered by female lay health workers to improve access to evidence-based care for depression and other common mental disorders through the Friendship Bench Project in Zimbabwe. Int J Ment Health Syst 2016;10:1-8.

17. Zimbabwe National Statistics Agency (ZIMSTAT) and ICF International. Zimbabwe demographic and health survey. Calverton, Maryland: ZIMSTAT and ICF International Inc, 2012.

18. Milgrom J, Gemmill AW, Bilszta JL, et al. Antenatal risk factors for postnatal depression: a large prospective study. J Affect Disord 2008;108(1-2):147-57.

19. Thase ME. Recommendations for Screening for Depression in Adults. JAMA 2016;315:349-50. 
20. Kroenke K, Spitzer RL, Williams JB. The PHQ-9: validity of a brief depression severity measure. J Gen Intern Med 2001;16:606-13.

21. Natamba BK, Achan J, Arbach A, et al. Reliability and validity of the center for epidemiologic studies-depression scale in screening for depression among HIV-infected and -uninfected pregnant women attending antenatal services in northern Uganda: a cross-sectional study. BMC Psychiatry 2014;14:303. A.

22. DeLong ER, DeLong DM, Clarke-Pearson DL. Comparing the areas under two or more correlated receiver operating characteristic curves: a nonparametric approach. Biometrics 1988;44:837-45. 